\title{
Using a serious game to assess spatial memory in children and adults
}

\author{
Mauricio Loachamín-Valencia ${ }^{1}$, M.-Carmen Juan ${ }^{1}(\bowtie)$, Magdalena Méndez-López ${ }^{2}$, \\ and Elena Pérez-Hernández ${ }^{3}$ \\ ${ }^{1}$ Instituto Universitario de Automática e Informática Industrial. Universitat Politècnica de \\ València. C/ Camino de Vera, s/n. 46022-Valencia, Spain \\ \{mrenan, mcarmen\}dsic.upv.es \\ ${ }^{2}$ Departamento de Psicología y Sociología. Universidad de Zaragoza. Zaragoza, Spain \\ mmendez@unizar.es \\ ${ }^{3}$ Departamento de Psicología Evolutiva y de la Educación. Universidad Autónoma de Ma- \\ drid. Madrid, Spain \\ elena.perezh@uam.es
}

\begin{abstract}
Short-term spatial memory has traditionally been assessed using visual stimuli, but not auditory stimuli. In this paper, we design and test a serious game with auditory stimuli for assessing short-term spatial memory. The interaction is achieved by gestures (by raising your arms). The auditory stimuli are emitted by smart devices placed at different locations. A total of 70 participants ( 32 children and 38 adults) took part in the study. The outcomes obtained with our game were compared with traditional methods. The results indicated that the outcomes in the game for the adults were significantly greater than those obtained by the children. This result is consistent with the assumption that the ability of humans increases continuously during maturation. Correlations were found between our game and traditional methods, suggesting its validity for assessing spatial memory. The results indicate that both groups easily learn how to perform the task and are good at recalling the locations of sounds emitted from different positions. With regard to satisfaction with our game, the mean scores of the children were higher for nearly all of the questions. The mean scores for all of the questions, except one, were greater than 4 on a scale from 1 to 5 . These results show the satisfaction of the participants with our game. The results suggest that our game promotes engagement and allows the assessment of spatial memory in an ecological way.
\end{abstract}

Keywords: Gamification, Serious Game, Auditory, Short-Term Memory, Karotz, color-depth sensor, Microsoft Kinect ${ }^{\mathrm{TM}}$, Natural User Interface.

\section{Introduction}

The terms gamification or serious games are commonly used interchangeably to refer to the use of games in non-game contexts. According to Deterding et al. [1], gamification refers to the use of design element characteristics for games in non-game contexts. 
The underlying idea of serious games is to combine game playing with a serious purpose (i.e., cognitive testing) [2]. Several works have suggested that serious games help in the learning process when they are used in relevant contexts that engage learners (e.g., [3]). For example, serious games have also been previously used for individuals with autism for learning purposes [4]; as an assessment tool for cognition and performance in an activity of daily living (in their case, cooking); or for training of cognitive functions in older adults [5].

Psychological science is interested in the assessment of a human's ability to learn about spatial and auditory information in different situations and contexts. Memory can be divided into short-term memory and long-term memory, depending on whether the information to be stored is useful for a limited period of time or is relevant to be stored stably for any future need [6]. Spatial and auditory memories have the capacity to store representations of spatial and auditory stimuli, respectively. Spatial memory allows us to find a place that we have visited before, follow a route after consulting a map, or remember where we left our belongings, among other examples. The assessment of spatial memory contributes to the understanding of individual differences in behavior and helps to prevent and detect pathology [6]. From a psychological perspective, there has been research interest in the ability to learn spatial and auditory information. The reason is that they are significant processes in daily life. Spatial learning is associated with academic outcomes [7] and with the functional maturation of the frontal pole [8]. Auditory learning facilitates taking notes while listening, written expression, and oral expression [9]. Some learning and behavioral problems are related to impairments in these processes $[10,11]$. Also, many learning experiences require considering auditory and spatial information simultaneously. Some examples are orientation in space and identification of people or objects. Since there are no procedures for assessing spatial learning for auditory stimuli, its implications for different types of learning remain unclear.

To our knowledge, the combination of Natural User Interfaces (NUI) and smart devices has not been explored for the assessment of cognitive processes, and especially for the assessment of spatial memory using auditory stimuli. We believe this combination promotes engagement and allows assessment in an ecological way. The development of new games for neuropsychological assessment represents an alternative for the evaluation of memory. These tools can be used for assessment as well as for training.

The objectives of our work are the following: to develop a game that is able to recognize gestures and integrate gesture recognition with smart devices; to obtain indicators of the participants' performance; to compare the performance obtained by using the game between the two groups of the study (i.e., children and adults); to compare the outcomes obtained on a questionnaire about perceptions and satisfaction and between the two groups.

A sample composed of adult participants is considered to determine the maximum performance with the game. We compare the performance between adults and children in order to determine whether or not the children achieve full competence in the game. Therefore, our research questions are the following: RQ1) Is the new game a valid tool for assessing spatial memory?; RQ2) Are the performance outcomes obtained with the 
game between children and adults different?; RQ3) Is the level of satisfaction with the game between children and adults different?

In order to answer the research questions we proceed as follows: RQ1) We observe the correlations between the outcomes obtained in the game and traditional tools; RQ2) We check if there are statistically significant differences in the performance outcomes obtained with the game between children and adults; RQ3) We check if there are statistically significant differences in the level of satisfaction between children and adults.

\section{Background}

\subsection{Gesture interaction}

Human body motion and gesture recognition have received increasing attention (e.g., $[12,13])$. Since the arms and, in particular, the hands are used to gesture and are a natural means of communication among humans, they are also of importance in $\mathrm{Hu}-$ man-Computer Interaction. Arm-motion recognition has been achieved through sensorbased and vision-based techniques. For sensor-based recognition, different sensors have been used to capture the position and orientation of the arms (e.g., accelerometer [14], or sensors worn on the body [15). The vision-based methods use images obtained through cameras/sensors, extract their characteristics, and analyze the actions performed by users. Recognition can be static or dynamic. Static gestures are time independent, whereas dynamic gestures are time dependent. The cameras/sensors can be of different types, color cameras (RGB), or color-depth cameras (RGB-D). Pisharady \& Saerbeck, 2015 [13] reviewed conventional hand-gesture recognition using RGB cameras as well as recognition using RGB-D sensors. Pisharady \& Saerbeck, 2015 [13] classified the techniques used for dynamic hand gesture recognition as: (a) Hidde Markov Models (e.g., [16]) and other statistical methods (e.g., [17]); (b) Artificial Neural Networks (e.g., [18]) and other learning based methods (e.g., [19]); (c) Eigenspacebased methods (e.g, [20]); (d) Curve fitting [21]; and (e) Dynamic programming [22]/Dynamic time warping (e.g., [23]). Depth sensors have already been used in computer vision for many years both commercial and non-commercial (e.g., [24]). An example of a non-commercial depth sensor is an IR Time-of-Flight Range Camera [24]. However, the appearance of low-cost, color-depth cameras/sensors led to a much more widespread use than their predecessors. Two of these sensors were Kinect ${ }^{\mathrm{TM}}[25,26]$ by Microsoft, and Xtion PRO LIVE by ASUS. Sensors of this type provide reliable tracking of human body postures and obtain the coordinates of a skeletal model. These coordinates can be used for human body motion and gesture recognition.

Low-cost, color-depth sensors have been used extensively for gesture interaction, and they have contributed to different areas. One of these areas is serious games. For example, Martín-SanJosé et al. [27] presented a game for learning historical ages. The researchers proposed a custom-built touch table and used the Microsoft Kinect ${ }^{\mathrm{TM}}$ sensor for hand-gesture recognition. They used this table to compare a personalized, freelearning itinerary with a linear learning itinerary. Their results showed that there were no statistically significant differences between the two learning itineraries. In another 
game for the same purpose $[28,29]$, this same group used Kinect ${ }^{\mathrm{TM}}$ for gesture interaction and autostereoscopic display for 3D perception. The interaction of the children was similar to the interaction that is used in our game. In their game, the children had to raise their hands to select the elements that appeared on the screen. In our case, the children also have to raise their hands. The main difference is that, in our case, the children did not have to select elements, just raise both arms. A similar proposal was also used for dental learning [30]. Homer et al. [31] determined the effects of interactivity in a Kinect-based literacy game for beginning readers. Those authors concluded that the activities in the game were not distracting. They were interesting and engaging activities for children, and they could support children's acquisition of language and literacy. Lin et al. [32] presented a game for a child to play blocks in a natural and intuitive way. They concluded that the users (children and adults) could fully immerse themselves in the game and construct a complicated structure easily. The game facilitated the learning experience.

With regard to how gesture interaction can assist in populations with special needs, one of the biggest contributions of low-cost, color-depth sensors is related to improvement in sign-language recognition. Sun et al. [33] proposed a discriminative exemplar coding for American sign-language recognition. Lee et al [34] also proposed a system for Taiwanese sign-language recognition that showed a good recognition rate. The proposals demonstrated feasibility and effectiveness. Armin et al. [35] reviewed the potential offered by low-cost, color-depth sensors in the context of educational methods for teaching children with sensory disabilities. For example, a Kinect-based game could help blind children to learn the name of objects by establishing links between tactile information and sound information. The authors highlighted the usefulness of low-cost, color-depth sensors as an attractive learning technology.

There are also games for the physical training of motor skills in children with a developmental disorder using color-depth sensors (e.g. autism, attention deficit/hyperactivity disorder, etc.), e.g., [36]. One of these games consisted of moving a girl along a path by using the movement of a hand [36]. The girl was shown on a TV screen or projector. The game aimed to train children in an engaging way and tried to keep their attention during the session. The game was compared with a classical procedure. There were significant improvements in motor learning of students with motor difficulties when the developed game was used. Similarly, another game showed positive effects for motor rehabilitation of children with cerebral palsy [37].

\subsection{Smart Devices}

Smart devices, especially those with a human appearance, have contributed to the study of human learning. One of the most interesting examples of their contributions is related to the field of autism. Adolescents with a diagnosis of autism played a face-match card game using a humanoid robot [38]. They played in pairs with a partner of a similar age who had a physical impairment. The game consisted of a face match. There were three playing conditions to establish comparisons: playing with the robot; playing with a computerized touch-screen whiteboard; and playing with conventional cards. Although the results were variable, they showed the feasibility of using robots in a school setting. 
Humanoid robots were also used for verbal learning. Children could learn words for several real objects in their first language after watching a video of a robot naming them [39]. Even toddlers aged 18-24 months could learn several words from a robot [40]. Robots have also been considered for learning about geometry in preschool education [41]. Children interacted with a Linux-based robot in the context of an educational game. The results showed positive effects of the experience with the robot in the learning of geometry. In addition, the interaction with this robot contributed to the spatial learning of three-year-old children [42]. Timms [43] emphasized the importance of the physical embodiment of the devices used. However, other types of devices showed interesting findings. For example, children learned about natural environments with a handheld device shaped like a horn that was used during the exploration of a woodland [44]. The horn provided non-speech audio sounds related to ecological sounds and the children had to interpret their significance (e.g., a light sabre sound means photosynthesis). The learning experience stimulated creativity and imagination in the children.

To our knowledge, the only study that has used the Karotz robot was presented by de Graaf et al. [45]. De Graaf et al. [45] installed Karotz robots in the home of older people. The goal was to improve their health. The role of the Karotz robot was to work as a 'personal assistant' who was interested in their progress and gave recommendations, such as controlling their weight.

\subsection{Assessment of spatial memory}

The assessment of spatial memory using visual stimuli has been carried out using different types of applications. For example, ARSM Task [46, 47] is a mobile augmented reality game for assessing spatial memory in children. The game was tested in a room with a size of about $5 \mathrm{~m}^{2}$. Real boxes were placed in the room. Inside the boxes were image targets and the virtual objects appeared on these image targets. The game consisted of 7 levels, in which the number of boxes and virtual objects to remember increased (in Level I, there were 2 boxes and 1 object to remember; in Level II, there were four boxes and 2 objects to remember, etc.). Juan et al. [46] found similarities in the results using ARSM Task and traditional methods. MnemoCity Task [48] had a virtual reality environment, with passive stereoscopy and natural interaction to evaluate spatial memory in children. The study compared two types of interaction: 1) a gamepad; 2) a steering wheel (with a built-in Wii Remote ${ }^{\mathrm{TM}}$ control) and Wii Balance Board ${ }^{\mathrm{TM}}$. The steering wheel was used to determine the user's turns. The Wii Balance Board ${ }^{\mathrm{TM}}$ was used to determine the participant's speed. The virtual environment recreated a park in which some tables were placed. The elements that the user had to locate appeared on the tables. Rodríguez et al. [48] also obtained correlations between their MnemoCity Task and traditional methods. Cárdenas et al. [49] developed a virtual labyrinth to assess spatial memory in adults. In this virtual labyrinth, the participant had to remember the route in order to find the exit. In their study, two types of interaction were compared: a gamepad and a bicycle. Pedaling on the bike indicated the speed. The handlebar turns indicated the turns that the avatar had to make in the virtual world. The performance of the participants that was obtained with the labyrinth correlated with traditional methods. 
There are several reasons for researchers to use gamification in the field of psychological assessment. The review of Lumsden et al. [50] described these reasons after considering empirical studies that tested the possibilities of games applied to the training or assessment of cognitive skills. These games were developed for processes such as executive functions and memory, combining different processes in certain cases. Lumsden et al. [50] mentioned some of the positive aspects that are related to the purpose of the assessment which include the following: the active involvement of the user and motivation; clearly determined goals; and the reduction of anxiety suffered during a testing session. This last aspect is very important for assessment in psychology, both for clinical and non-clinical populations.

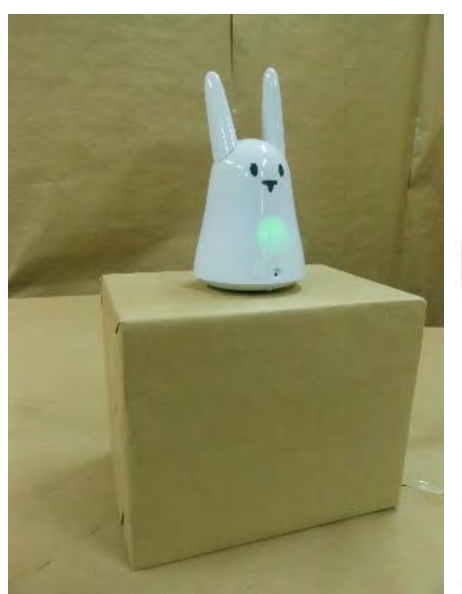

Fig. 1. Karotz rabbit.

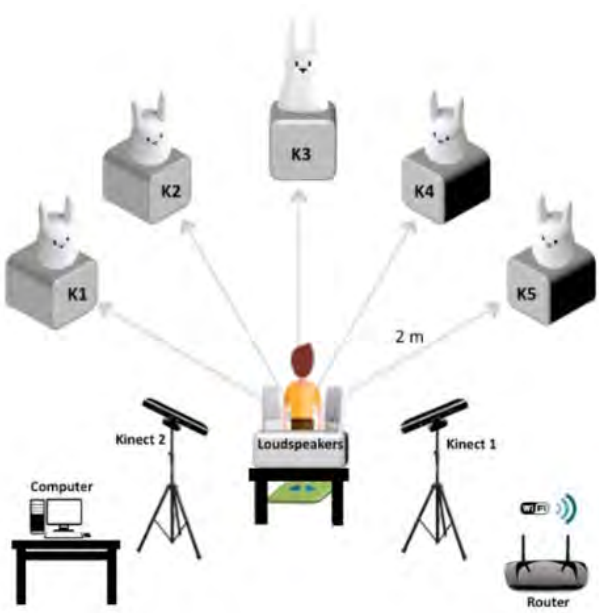

Fig. 2. Diagram of the game area.

\section{Design and development of the game}

\subsection{Design of the game}

The game was designed to use Natural User Interfaces (NUI) and smart devices for managing auditory stimuli. For the NUI, the proposal was to use a low-cost, color-depth sensor to capture movements. We used Microsoft Kinect ${ }^{\mathrm{TM}}$, but other sensors could also be used. Karotz rabbits were proposed as smart devices. Fig. 1 shows an image of a Karotz rabbit. The game was designed to test the users' ability to detect and localize auditory stimuli that are emitted in different positions of a game area (Fig. 2). The game consists of guessing the rabbit that emitted a sound. However, the rabbits are identical. The only difference is their performance (sounds and movements of their ears). Therefore, the player must concentrate on the rabbits' locations and memorize them. Some communication codes were identified and defined. When a player raises his/her arms in front of a rabbit, it means "Hello, I know you did it". The rabbits move their ears and turn on lights when they want to get the attention of the player (which means "Hello, 
I'm here.") or when they have understood the player's response (which means "Agreed!"). The children are told that they have to hear the response of the Karotz when they raise their arms in order to be sure that the Karotz has understood their action. If this response is not emitted, the children must repeat the action. Fig. 3 shows a participant that is raising his arms in front of a Karotz rabbit.

Our game is framed in the short-term type of memory. We are interested in the ability of the participants to retrieve a sequence of locations of the auditory stimuli emitted. This sequence is also called "memory span", which represents the capacity of shortterm storage to retain spatial items. We are interested in determining the maximum capacity of each participant in this type of memory span. A participant that did not retrieve a certain memory span in a certain number of attempts would not have been able to retrieve a longer memory span. Based on our experience, we have established that the number of attempts in our game in each level is 3 .

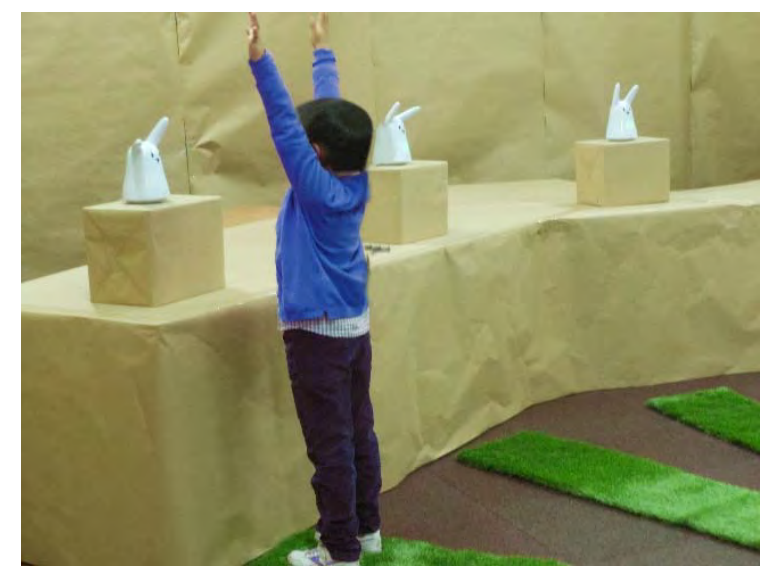

Fig. 3. A participant raising his arms in front of a Karotz rabbit.

Fifteen children participated in a preliminary study to determine which gestures were easier for Kinect ${ }^{\mathrm{TM}}$ to recognize. It was determined that the most appropriate gesture for our game was to raise both arms at the same time in front of a Karotz. The identification was correct $95 \%$ of the time (when raising the arms and then lowering them immediately). To achieve $100 \%$ detection, the children were told to stand in front of a Karotz with their arms raised until they heard the message from the Karotz, which indicated that the Karotz had been selected ("Agreed!").

The area of the game for our study was defined to be around $5 \mathrm{~m}^{2}$. Nevertheless, this area can be delimited within a room with larger dimensions. In our study, artificial turf was placed on the floor for guide paths, and a rug was used to indicate the initial position of the player. The walls were covered with wrapping paper to eliminate any spatial cues. The height of the Karotz rabbit was adjusted by placing cardboard boxes on the tables. Five Karotz rabbits were placed on tables with the following locations (see Fig. 2): Karotz rabbit $1\left(-60^{\circ}\right)$, Karotz rabbit $2\left(-30^{\circ}\right)$, Karotz rabbit $3\left(0^{\circ}\right)$, Karotz rabbit 4 $\left(+30^{\circ}\right)$, Karotz rabbit $5\left(+60^{\circ}\right)$. Each Karotz rabbit emits its assigned sound. The game 
included a total of 45 acoustic stimuli, which should be randomly emitted in different locations to avoid repetitions or established sequences. The game was defined to be composed of five different levels based on the number of stimuli presented in each trial (Fig. 4). Each level was defined to relate to a specific theme. The chosen themes were: nature, a party, a farm, a house, and a big city. Each level consists of 3 trials. The difference between levels lies in the number of sounds to be used in each trial, which will increase by 1 at each subsequent level. Specifically, the acoustic stimuli were distributed as follows: Level I ( 1 acoustic stimulus for each trial, 3 stimuli in total); Level II ( 2 acoustic stimuli for each trial, 6 stimuli in total); Level III (3 acoustic stimuli for each trial, 9 stimuli in total); Level IV (4 acoustic stimuli for each trial, 12 stimuli in total); and Level V (5 acoustic stimuli for each trial, 15 stimuli in total).

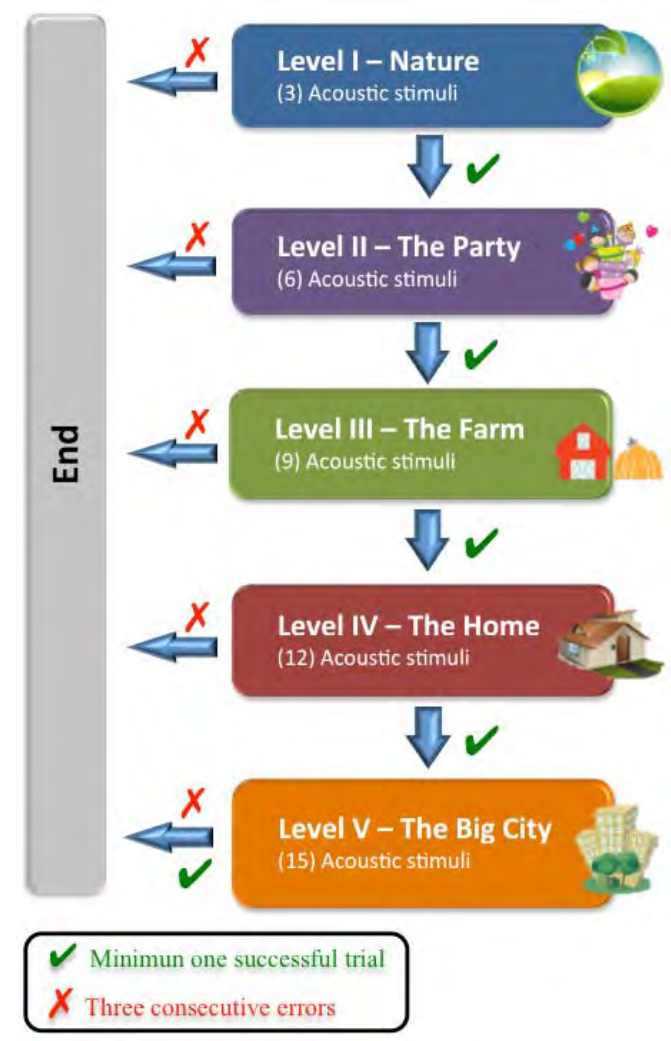

Fig. 4. Levels of the game.

Each level has two phases: the search phase and the location phase. In the search phase, the user learns the sounds and their location. First, the user listens to the instructions through the loudspeakers. Then, the user listens to the continuous sound emitted by a Karotz and memorizes its location. While the sound is playing, the participant moves to stand in front of the Karotz that emits the sound and raises his/her arms to make the 
selection. The Karotz emits a message to indicate that it has understood the gesture. The user is told that he/she must listen to the message of the Karotz after raising his/her arms. If the Karotz does not emit such a message, the user must repeat the action.

The participant's spatial memory is evaluated in the location phase. First, the user listens to the instructions through the loudspeakers and also the sound that has to be located. In this phase, the user has to remember the location of the Karotz that emitted the stimulus (sound). The stimuli are only emitted once. The user has to move to the correct location and raise his/her arms in front of the Karotz in order to select it. Then he/she must return to the starting position. The game stores all of the answers (successes or failures).

A trial is successfully passed if all of the sounds are correctly located. If a sound is not correctly located, the trial has not been passed. If there is at least one successful test of the 3 trials of a given level, the user has passed that level and advances to the next level. The participant must perform the 3 trials of one level, regardless of whether he/she has successfully passed all of the stimuli of the first trial. If the user fails in all 3 trials at any level, the game ends. The game also ends when the participant completes level V.

\subsection{Hardware and Software}

We used two Microsoft Kinect ${ }^{\mathrm{TM}} \mathrm{v} 1$ devices and five Karotz rabbits. The Kinect ${ }^{\mathrm{TM}} \mathrm{v} 1$ devices include a RGB camera with a resolution of $640 \times 480$ pixels, an infrared camera, an infrared projector, and a multiarray microphone. The Karotz rabbits are shaped like a rabbit and are $30 \mathrm{~cm}$ tall (Fig. 1). They can connect to the Internet through a wireless access point. They have loudspeakers, a webcam, an LED-light (in their bellies), and they can move their ears. Their technical specifications are: $400 \mathrm{MHz}$ ARMCPU, $64 \mathrm{MB}-\mathrm{RAM}, 256 \mathrm{MB}$ of storage, and a Linux operating system.

An HP computer with an Intel i5 processor and Windows 7 operating system was used. This computer had USB ports connected to a separate USB host controller. This allowed two Kinect ${ }^{\mathrm{TM}}$ devices to be used simultaneously. Additionally, this computer was used as the server. Two conventional loudspeakers were used to give instructions during the game. We used a wireless-G Router with WAN port for networking and accessing the Internet. This Internet access was required by the five Karotz rabbits and the computer.

The sounds were edited using Audacity 2.0.3 to ensure the loudness of $70 \mathrm{~dB}$, frequency $>3000 \mathrm{~Hz}, 4-\mathrm{sec}$ duration, and stereo format. For the voice of the messages, the audio clips were recorded using Audacity 2.0.3, and they had identical characteristics to the sounds, except that the duration varied depending on the specific instructions or message.

Visual Basic 2008 Express Edition was used for the development of the system that manages the procedure during the game and the graphical interface for the supervisor. To program the Kinect ${ }^{\mathrm{TM}}$ device, we used Visual C++ 2010 Express Edition, Kinect SDK 1.8, OpenNI 2.0 SDK, and Nite. The system has three modules: (a) one to configure and manage the Karotz rabbits, their IPs, and the IP of the sounds server; (b) one to 
register the participant's information and for the evaluation process; and (c) one to manage the communication among the Karotz rabbits and the Kinect ${ }^{\mathrm{TM}}$ devices.

The system has a graphical interface that allows the supervisor to introduce the player's code, date of birth, and gender. The role of the supervisor is to supervise the task by observing the supervisor's interface, which offers information about the participant's progress. The supervisor does not control the rabbits. The supervisor can observe the performance carried out by the player (i.e., trials, successes, and failures (Fig. 5)).

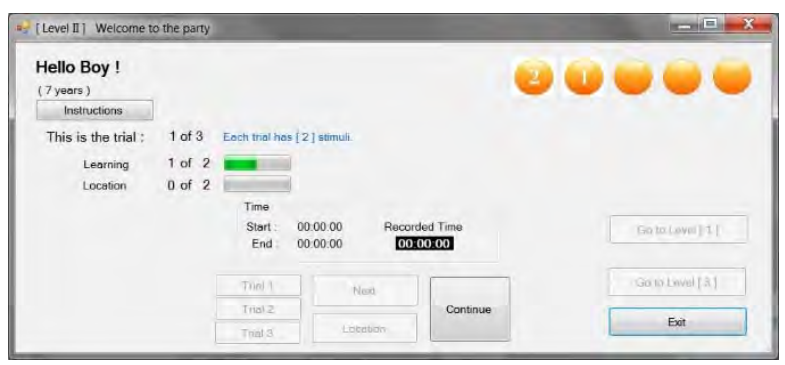

Fig. 5. Screen that displays the player's performance.

\section{Study}

\subsection{Participants}

A total of 70 participants were involved in our study. A total of 32 healthy children (16 girls, 16 boys) took part in this study, with ages ranging from 9 to 10 years old. The children were attending the fifth grade at a public school. Before carrying out the study, the parents of the participating children received written information about the aims and procedures of the study, and they signed a consent form to allow their children to participate. The children who participated received a diploma and a snack immediately after the test session. They were not informed about this reward until the end of the study.

A total of 38 healthy adults (19 women, 19 men) took part in the study, ranging in age between 18 and 28 years old $(\mathrm{M}=21.32, \mathrm{SD}=2.86)$. The participants were recruited throughout the campus of a large public university. The students had the following education levels: vocational education $(28.95 \%)$, undergraduate $(44.74 \%)$, or graduate $(26.31 \%)$. Before the study, all of the participants were informed in writing about the aims and procedures, and they signed an informed consent form. The participants received a diploma and a snack right after the test session, but they were not informed about the snack until they had completed the procedures of the study.

The study was conducted in accordance with the European Directive 2001/20/EC and the Helsinki Declaration for biomedical research involving humans. The Ethics Committee of the Universitat Politècnica de València (UPV) approved the research protocol. 


\subsection{Measurements}

We assessed the participants' ability to recall auditory stimuli and their locations by registering their performance during the task. The database of the system recorded successes or failures in the detection and location of stimuli. Four variables based on performance during the task were stored. These variables were the following: Number of Correct Stimuli (NCS), Number of Correct Levels (NCL), Task Time (TT), Number of Errors (NE). NCS was the number of auditory icons that a participant could successfully locate. NCL was the sum of the number of levels correctly performed. TT was the total time in seconds that a participant spent to complete the task. Finally, NE was the sum of the number of errors that a participant could commit. In addition, we calculated the task score (TS) which is the sum of all auditory icons (of any block and Level) for which the participant correctly indicated the emitting rabbit. The TS allows us to determine the performance of the participants. The maximum possible task score was 45 .

Visuospatial learning and auditory learning were also assessed in the participants by using traditional methods. We selected specific subtests that are included in the Test of Memory and Learning battery (TOMAL) [51]. The TOMAL battery assesses various domains of learning. We also selected subtests that are included in the EDAF test. The EDAF measures auditory and phonological discrimination [52]. We used the direct scores obtained in all of the subtests used.

We selected the TOMAL subtest for the assessment of visuospatial learning: Memory for Location (ML). This subtest consists of a spatial recall task of one or more large dots that appear within a square or rectangle. The participant is asked to identify the location of the dots within a grid. The range of the grid is $3 \times 3$ and $4 \times 4$ (with 9 locations and 16 locations, respectively). In order to assess immediate retrieval of auditory items, we used two verbal span subtests of the TOMAL battery: Digits Forward (DF) and Digits Backward (DB). The DF is a number recall task that measures lowlevel rote recall of a sequence of numbers. The DB task (a variation of the DF task) consists of a sequential recall of a sequence of numbers but in reverse order. For the assessment of auditory and phonological discrimination, we selected the EDAF subtest: Environmental Sound Discrimination (ESD). The ESD is a discrimination task about sounds of the environment that are played on a CD (i.e., baby's crying, traffic noise, etc.). We also measured the participants' everyday memory. We selected eight questions from the ECM-Questionnaire (ECM) for this purpose [53]. The skills are rated on a 4-point Likert scale $(1=$ never to $4=$ almost always). The questions are "I have good spatial orientation, I get lost where I have often been before, I forget where I have put things, I recognize the places I have been before, I know how to go home, I remember where I store my things, I get lost in familiar places, I forget how to go to a place that I have already been explained how to get to". In the case of the child participants, their parents completed the Parent Report version of this questionnaire.

To assess the participants' perceptions and satisfaction with the game, we designed a Questionnaire (QS) based on the questionnaires of Lewis [54] and Lund [55] (Table 1). Items were selected from these two existing instruments based on their appropriateness for assessing learning, satisfaction, and interaction with the game, and the items 
were adapted to our studies. The participants responded to the items using a 5-point Likert scale ( 1 = "strongly disagree" to $5=$ "strongly agree").

Table 1. Questionnaire (QS).

\begin{tabular}{cl}
\hline Question ID & \multicolumn{1}{c}{ Question } \\
\hline Q1 & It was easy to use this task \\
Q2 & It was easy to learn to use this task \\
Q3 & I would recommend it to a friend \\
Q4 & Overall, I am satisfied with this task \\
Q5 & The interactive interface is pleasant (body movements) \\
Q6 & I like using the auditory interface (sounds) \\
Q7 & Overall, this interaction was fun \\
\hline
\end{tabular}

\subsection{Study design}

For the children, the test sessions took place from Monday to Friday between 9:00 and 14:00 over three weeks during the normal school year. For the adults, the study was carried out over two weeks, from Monday to Friday between 9:00 and 15:00. A supervisor guided the participants through the steps to follow during testing and helped them become familiar with the area of interaction. The supervisor did not interfere with the game performance unless the participants requested assistance or experienced a technical problem (system failure). At the end of the session, the supervisor administered the QS questionnaire to each participant.

\subsection{Performance outcomes using the game}

Data from the children and adults were analyzed using the statistical open source toolkit $R$ (www.r-project.org) with the RStudio IDE Desktop (www.rstudio.com). The normality of the data was analyzed based on Shapiro-Wilk and Anderson-Darling tests. The TS, NCS, NCL, and NE variables did not fit the normal distribution, so we applied the Mann-Whitney $U$ test for unpaired data. The TT variable did fit the normal distribution, so an ANOVA test was used. The Mann-Whitney $U$ test for unpaired data was used to determine the statistically significant differences for the QS questionnaire between children and adults.

The results showed that the group of children performed worse than the group of adults (see Table 2). The Task Score (TS) indicates statistically significant differences in favor of the adults (Fig. 6). The results of the comparisons indicate statistically significant differences for the variables related to successes (NCS and NCL) in favor of the adults. Also, the group of children committed a significantly higher number of errors (NE) and they spent more time on the game (TT) than the adults. 
Table 2. Mann-Whitney $U$ test analysis for TS, $\mathrm{NCS}, \mathrm{NCL}_{2}$ and $\mathrm{NE}$ variables. ANOVA for the TT variable.

\begin{tabular}{cccccc}
\hline Var. & $\begin{array}{l}\text { Child } \\
\mu \pm \sigma\end{array}$ & $\begin{array}{l}\text { Adult } \\
\mu \pm \sigma\end{array}$ & $U$ & $Z$ & $p$ \\
\hline TS & $34.28 \pm 11.35$ & $43.68 \pm 1.97$ & 153.0 & -5.465 & $<0.001^{*}$ \\
NCS & $34.28 \pm 11.35$ & $43.68 \pm 1.97$ & 153.0 & -5.465 & $<0.001^{*}$ \\
NCL & $2.75 \pm 1.27$ & $4.29 \pm 1.01$ & 199.5 & -4.988 & $<0.001^{*}$ \\
NE & $4.53 \pm 0.98$ & $4.08 \pm 0.78$ & 849.0 & 3.117 & $<0.001^{*}$ \\
\hline \multirow{2}{*}{ Var. } & Child & Adult & d.f. & $F$ & $p$ \\
\hline TT & $\quad 610.2 \pm 165.5$ & $518.2 \pm 52.7$ & 1,68 & 10.49 & $0.002^{*}$ \\
\hline
\end{tabular}

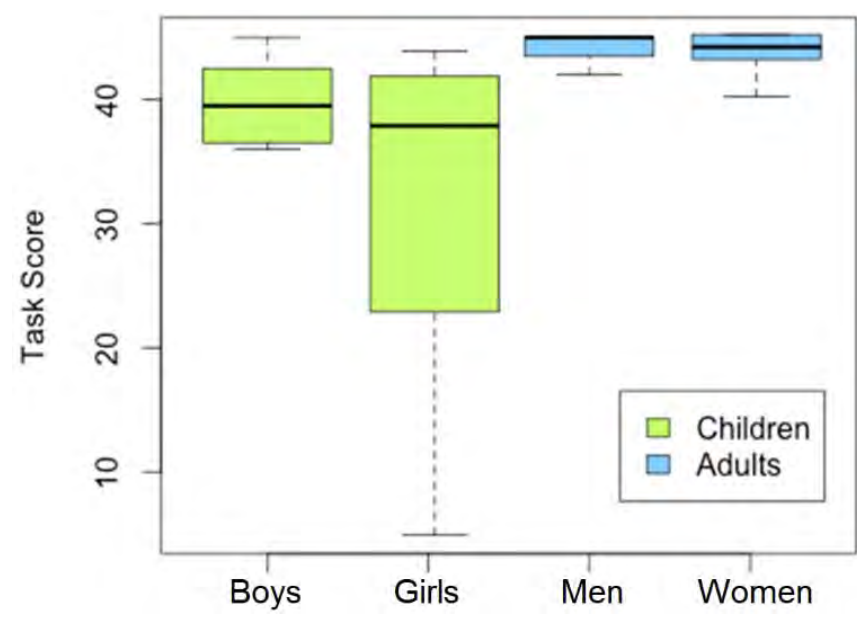

Fig. 6. Graph showing the outcomes for the TS variable.

\subsection{Outcomes using traditional methods}

The outcomes obtained with the traditional methods were analyzed to determine whether or not there were differences between the two groups. Table 3 shows the results and statistics. The results showed that adults demonstrated significantly greater visuospatial learning (DB, DF, ML and ECM). For the discrimination of sounds, there were no statistically significant differences for the ESD variable. 
Table 3. Mann-Whitney $U$ test analysis for DB, ESD, and ECM. ANOVA for the DF and ML variables.

\begin{tabular}{|c|c|c|c|c|c|}
\hline & $\begin{array}{l}\text { Child } \\
\mu \pm \sigma\end{array}$ & $\begin{array}{l}\text { Adult } \\
\mu \pm \sigma\end{array}$ & $U$ & $Z$ & $p$ \\
\hline DB & $20.66 \pm 8.43$ & $36.74 \pm 13.80$ & 144.5 & -5.47 & $<0.001^{*}$ \\
\hline ESD & $14.34 \pm 0.90$ & $14.66 \pm 0.53$ & 510.0 & -1.35 & 0.189 \\
\hline \multirow[t]{2}{*}{ ECM } & $3.62 \pm 2.77$ & $11.11 \pm 4.24$ & 71.5 & -6.34 & $<0.001^{*}$ \\
\hline & $\begin{array}{l}\text { Child } \\
\mu \pm \sigma\end{array}$ & $\begin{array}{l}\text { Adult } \\
\mu \pm \sigma \\
\end{array}$ & d.f. & $F$ & $p$ \\
\hline $\mathrm{DF}$ & $8.12 \pm 1.84$ & $57.13 \pm 14.86$ & 1,68 & -18.52 & $<0.001^{*}$ \\
\hline ML & $11.03 \pm 6.15$ & $19.05 \pm 5.11$ & 1,68 & -5.96 & $<0.001^{*}$ \\
\hline
\end{tabular}

\subsection{Correlations between our game and traditional methods}

In order to compare the participants' performance when using the game (TS) and traditional methods (DB, DF, ML, ESD, and ECM), correlations were calculated with the complete sample ( 32 children and 38 adults). The Spearman correlation was applied and the effect size of the correlation was obtained, rho $(\rho)$. The correlations between the TS variable and the traditional method variables are: $\operatorname{ML}(\rho=0.43, p<0.001 *)$ and DB $(\rho=0.45, p<0.001)$. All correlations are linear and positive. These correlations demonstrate the similarity between our game and those traditional methods (ML and DB).

\subsection{User experience}

Table 4 shows the results of the statistical analysis applied to the answers to the QS questionnaire about the users' experience and the comparison of the scores between children and adults. Both groups found the game easy to use and easy to learn (Q1 and Q2). The children were significantly more likely than the adults to recommend the game to their friends (Q3), and they were significantly more satisfied with the game (Q4). Both groups found the interface pleasant to use with regard to body movements (Q5) and the auditory interface (Q6). The children found the interface significantly more fun than the adults (Q7) (Table 4).

Table 4. Mann-Whitney $U$ test analysis and $r$ effect size for differences between children and adults on the QS questionnaire. '**' indicates significant difference at level $\alpha=0.05$.

\begin{tabular}{ccccccccc}
\hline Q\#. & Children & $\mu_{\mathrm{CH}} \pm \sigma_{\mathrm{CH}}$ & Adults & $\mu_{\mathrm{A}} \pm \sigma_{\mathrm{A}}$ & $U$ & $Z$ & $p$ & $r$ \\
\hline 1 & {$[5] ;[1]$} & $4.44 \pm 0.88$ & {$[4] ;[1]$} & $4.21 \pm 0.70$ & 748.5 & 1.832 & 0.069 & 0.219 \\
2 & {$[5] ;[0]$} & $4.81 \pm 0.40$ & {$[5] ;[0]$} & $4.82 \pm 0.39$ & 606.0 & -0.035 & $>0.99$ & 0.004 \\
3 & {$[5] ;[0]$} & $4.53 \pm 0.98$ & {$[4] ;[1]$} & $4.08 \pm 0.78$ & 849.0 & 3.117 & $<0.001^{* *}$ & 0.373 \\
4 & {$[5] ;[1]$} & $4.62 \pm 0.55$ & {$[4] ;[1]$} & $4.32 \pm 0.62$ & 772.0 & 2.182 & $0.035^{* *}$ & 0.261 \\
5 & {$[4] ;[2]$} & $4.09 \pm 1.06$ & {$[4] ;[2]$} & $3.89 \pm 0.92$ & 696.5 & 1.105 & 0.278 & 0.132 \\
6 & {$[4] ;[1]$} & $4.25 \pm 0.80$ & {$[4] ;[1]$} & $4.29 \pm 0.69$ & 605.5 & -0.032 & 0.997 & 0.004 \\
7 & {$[5] ;[0]$} & $4.78 \pm 0.49$ & {$[4] ;[1]$} & $4.24 \pm 0.54$ & 918.5 & 4.162 & $<0.001^{* *}$ & 0.497 \\
\hline
\end{tabular}


Fig. 7 shows graphically the mean scores for the QS questionnaire. The children gave a mean score of $4.55 \pm 0.55$ on the QS questionnaire, and the adults gave a mean score of $4.26 \pm 0.47$. These results and the observations of the supervisor indicate that both children and adults understood how to interact with the game and were able to learn and recall a sequence of auditory icons with their respective locations while moving.

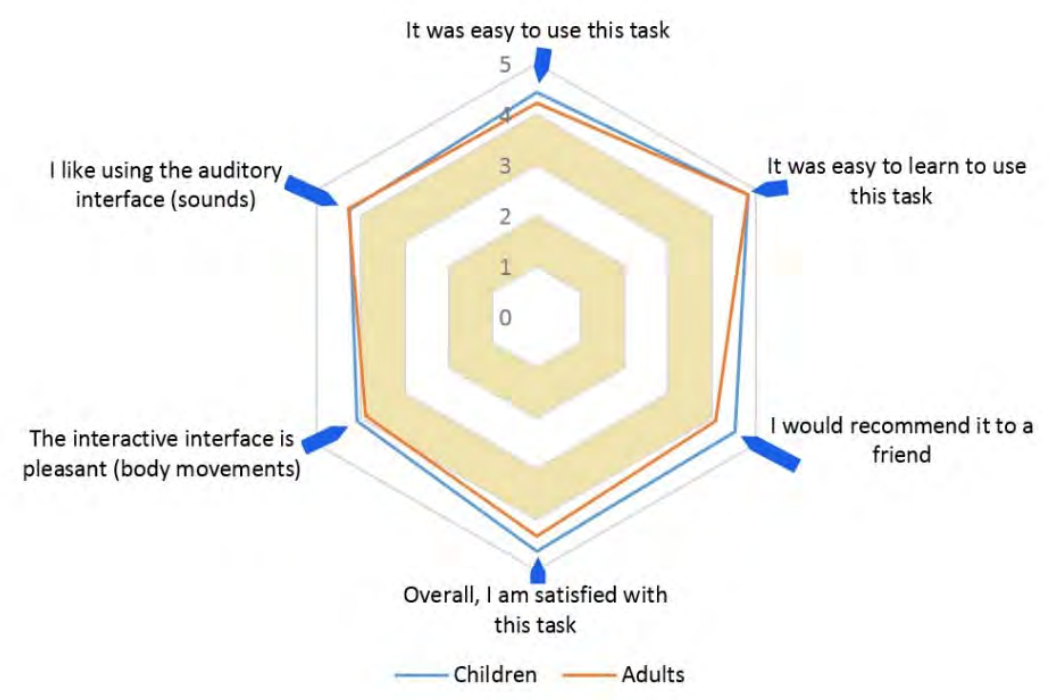

Fig. 7. Graph showing the mean scores for the questionnaire.

\section{Discussion}

Our game combines Natural User Interfaces (using gestures) with smart devices. The game was designed for the assessment of spatial memory by using auditory stimuli. Previous proposals for the assessment of spatial memory are centered on visual cues [46-49]. Traditional methods consider each skill separately [51-53]. However, our game combines auditory stimuli with real visual cues for the assessment of spatial memory.

A study involving 70 participants (32 children and 38 adults) was carried out. The participants played with our game, and traditional tests [51-53] were also administered. The results of the game were compared with the results obtained using traditional methods. Correlations were found between our game and traditional methods, indicating that our game has proven to be a valid tool for assessing spatial memory by using auditory stimuli for both children and adults. This result affirmatively answers our first research question (RQ1). These correlations are in line with previous works for assessing spatial memory (only based in visual cues) that also obtained correlations between their tasks and traditional methods [46-49]. This result is also in line with other previous works 
that have demonstrated that serious games have the potential to be used as assessment tools (e.g., [56]) or for diagnosis (e.g., [57]).

With regard to ecological validity, in neuropsychological assessment, ecological validity can be defined as the "functional and predictive relation between the participant's performance on a set of neuropsychological tests and the participant's performance in a variety of real-world settings" [58]. Therefore, the development of new games for neuropsychological assessment represents an important tool for early identification of atypical development or for assessment of memory as a cognitive function that is linked to various intellectual and social activities of children and adults. Games of this type could contribute to a better understanding of the influence of different variables on the cognitive development of children and adults. Our game can be used for assessing cognitive processes in an ecological way. Our game is in line with previous serious games that have shown ecological validity [59]. However, we have not checked the influence that the ecological component has on the participants. A future study could determine the advantages and disadvantages of our proposal with respect to another modality that does not have the ecological component.

The performance outcomes with the game were significantly lower for the children. This result answers our second research question (RQ2) indicating that the age range of the participating children (9-10 years old) did not achieve full competence in the game. The game requires constant attention and concentration since it involves the simultaneous evaluation of spatial and auditory abilities. The fact that the results were better for adults is consistent with the idea that adults can store more elements in shortterm memory than children because the ability of the humans increases continuously during maturation [6].

With regard to perceptions and satisfaction with the game, the mean scores for all questions for both the children and the adults were greater than 4 (on a scale of 1 to 5). Only Q5 for the adults had a mean score of 3.89 ("Q5: The interactive interface is pleasant (body movements)"). Therefore, we can conclude that all of the participants found the game easy to learn and easy to use, they had a good time, and they have shown their satisfaction with the game. The analysis showed that the children scored significantly higher on questions Q3 (recommend the game to a friend), Q4 (satisfied with the game), and Q7 (fun). This result answers our third research question (RQ3) indicating that the children were significantly more satisfied with the game than the adults. Since the game seemed easy to use for both children and adults, they were able to concentrate on the tasks to be done rather than on the control mechanisms. The Karotz make sounds and move their ears and illuminate their central part, so the participants can consider them as pets or toys. This aspect could influence the greater fun experienced by the children. The use of multiple sensory modalities may influence participants' satisfaction. The communication of the participants with the Karotz raising their arms can be interpreted as a code of communication between equals, which can influence the attractiveness of the game. Many participants were accustomed to this type of interaction given the proliferation of games that use color-depth sensors, so this type of interaction seemed natural. From our point of view, all of this contributes to the immersion that our game induces and the motivation that the participants showed. The supervisor's observations corroborate our perception of immersion. The supervisor 
added that many participants did not realize that the Karotz rabbits were there and they even talked to the Karotz rabbits. However, we have not yet determined the influence that the physical appearance of the Karotz had on the children and their motivation for the activity. The study of this influence could be part of another work.

With regard to the interaction and selection of the Karotz, in this work the interaction has been achieved through gestures. However, the button that the Karotz have on top could be used, a natural language user interface could be used, the correct Karotz could be selected on a tablet, etc. As future work, we are studying the incorporation of other types of interaction and their comparison. However, the intention of our proposal is for the game to be used by blind people and the alternative interaction would also have to consider this population. Thus, pressing the button on the top of the Karotz or using a tablet would not be viable alternatives. Also, other types of robots that are currently on the market or that could appear in the future could be used to reproduce or improve our proposal.

\section{Conclusion}

Our game presents an alternative tool to assess both multimodal integration and learning of auditory and spatial information in children and adults. The game could be used for assessment and training of spatial memory. The game could help in the identification of alterations in spatial memory in both children and adults. In children, the game could help in the early identification of atypical development, children with Attention Deficit Hyperactivity Disorder, etc. In adults, the game could help in the identification of alterations in memory, such as Alzheimer's disease, other types of dementia with alterations of orientation, patients with sequelae after suffering a stroke or head trauma, etc.

As a computer-based game, our game facilitates the control of the presentation of stimuli and the recording of responses. We assume that a supervisor of this experience might make some mistakes due to distraction and/or tiredness, among other causes. The game could be enhanced by adding visual keys (lights in the bellies of the Karotz rabbits or movement of their ears). In this paper, we have compared children and adults, but other comparisons are also possible. For example, it would be interesting to compare the performance of children with normal vision and blind children or to use this new game with children with developmental disorders. Another study could focus on the suitability of the game for people with autism. In that study, it would be very interesting to determine if the appearance of the Karotz is especially suitable for this population.

\section{Acknowledgments}

This work was mainly funded by the Spanish Ministry of Economy and Competitiveness (MINECO) through the CHILDMNEMOS project (TIN2012-37381-C02-01) and cofinanced by the European Regional Development Fund (FEDER).

Other financial support was received from the Government of the Republic of Ecuador through the Scholarship Program of the Secretary of Higher Education, Science, 
Technology and Innovation (SENESCYT), the Conselleria d'Educació, Investigació, Cultura i Esport through the grant for consolidable research groups in favour of the Computer Graphics and Multimedia group of the ai2 (PI. Prof. M.-Carmen Juan) (20172018), the Government of Aragon (Department of Industry and Innovation), and the European Social Fund for Aragon.

We would like to thank the following for their contributions: Jimena Bonilla; the users who participated in the study; and the reviewers for their valuable comments.

\section{References}

1. Deterding, S., Dixon, D., Khaled, R., Nacke, L.: From game design elements to gamefulness: Defining "Gamification". In: Proceedings of the 15th International Academic MindTrek Conference: Envisioning Future Media Environments (MindTrek'11). pp. 9-15. ACM New York, NY, USA (2011).

2. Wiemeyer, J., Kliem, A.: Serious games in prevention and rehabilitation - a new panacea for elderly people?. Eur. Rev. Aging Phys. Act. 9, 41-50 (2012).

3. Catalano, C.E., Luccini, A.M., Mortara, M.: Best practices for effective design and evaluation of serious games. International Journal of Serious Games. 1, e1-e13 (2014).

4. Whyte, E.M., Smyth, J.M., Scherf, K.S.: Designing serious game interventions for individuals with Autism. J. Autism Dev. Disord. 45, 3820-3831 (2015).

5. Kim, K.-W., Choi, Y., You, H., Na, D.L., Yoh, M.-S., Park, J.-K., Seo, J.-H., Ko, M.-H.: Effects of a serious game training on cognitive functions in older adults. J. Am. Geriatr. Soc. 63, 603-605 (2015).

6. Lezak, M.D.: Neuropsychological assessment. Oxford University Press, New York, NY (1995).

7. Rourke, B.P.: Arithmetic disabilities, specific and otherwise: A neuropsychological perspective. J. Learn. Disabil. 26, 214-226 (1993).

8. Arai, S., Okamoto, Y., Fujioka, T., Inohara, K., Ishitobi, M., Matsumura, Y., Jung, M., Kawamura, K., Takiguchi, S., Tomoda, A., Wada, Y., Hiratani, M., Matsuura, N., Kosaka, H.: Altered frontal pole development affects self-generated spatial working memory in ADHD. Brain Dev. 38, 471-480 (2016).

9. Dehn, M.J.: Cognitive Processing Deficits. In: Morris, R.J. and Mather, N. (eds.) Evidence-Based Interventions for Students with Learning and Behavioral Challenges. pp. 258-287. Routledge, New York and London (2008).

10. Graham, J.A., Heywood, S.: The effects of elimination of hand gestures and of verbal codability on speech performance. Eur. J. Soc. Psychol. 5, 189-195 (1975).

11. Rauscher, F.H., Krauss, R.M., Chen, Y.: Gesture, Speech, and Lexical Access: The Role of Lexical Movements in Speech Production. Psychol. Sci. 7, 226-231 (1996).

12. Khan, R.Z., Ibraheem, N.A.: Hand Gesture Recognition: A Literature Review. International Journal of Artificial Intelligence \& Applications. 3(4), 161-174 (2012).

13. Pisharady, P.K., Saerbeck, M.: Recent methods and databases in vision-based hand gesture recognition: A review. Computer Vision and Image Understanding. 141, 152-165 (2015).

14. Agrawal, S., Constandache, I., Gaonkar, S., Choudhury, R.R., Caves, K., DeRuyter, F.: Using mobile phones to write in air. In: Proceedings of the 7th ACM International Conference on Mobile Systems, Applications, and Services. pp. 15-28. Washington, DC, USA (2011). 
15. Park, T., Lee, J., Hwang, I., Yoo, C., Nachman, L., Song, J.: Egesture: a collaborative architecture for energy-efficient gesture recognition with hand-worn sensor and mobile devices. In: Proceedings of the 9th ACMConference on Embedded Networked Sensor Systems. pp. 260-273. ACM, Seattle, Wash, USA (2011).

16. Beh, J., Han, D.K., Durasiwami, R., Ko, H.: Hidden Markov model on a unit hyper-sphere space for gesture trajectory recognition. Pattern Recognit. Lett. 36, 144-153 (2014).

17. Suk, H.I., Sin, B.K., Lee, S.W.: Hand gesture recognition based on dynamic Bayesian network framework. Pattern Recognit. 43(9), 3059-3072 (2010).

18. Yang, M.H., Ahuja, N., Tabb, M.: Extraction of 2D motion trajectories and its application to hand gesture recognition. IEEE Trans. Pattern Anal. Mach. Intell. 24(8), 1061-1074 (2002).

19. Shen, X.H., Hua, G., Williams, L., Wu, Y.: Dynamic hand gesture recognition: an exemplar-based approach from motion divergence fields. Image Vis. Comput. 30(3), 227-235 (2012).

20. Patwardhan, K.S., Roy, S.D.: Hand gesture modelling and recognition involving changing shapes and trajectories, using a predictive eigentracker. Pattern Recognit. Lett. 28, 329334 (2007).

21. Shin, M.C., Tsap, L.V., Goldgof, D.B.: Gesture recognition using Bezier curves for visualization navigation from registered 3-D data. Pattern Recognit. 37(5), 1011-1024 (2004).

22. Kuremoto, T., Kinoshita, Y., Feng, L., Watanabe, S., Kobayashi, K., Obayashi, M.: A gesture recognition system with retina-v1 model and one-pass dynamic programming. Neurocomputing. 116, 291-300 (2012).

23. Corradini, A.: Dynamic time warping for off-line recognition of a small gesture vocabulary. In: Proceedings of the IEEE International Workshop on Computer Vision (ICCVW 2001). pp. 82-89. IEEE (2001).

24. Breuer, P., Eckes, C., Müller, S. tefan. 2007. Hand Gesture Recognition with a Novel IR Time-of-Flight Range Camera-A Pilot Study. In: Proceedings of the 3rd International Conference on Computer Vision/Computer Graphics Collaboration Techniques. pp. 247-260 Springer-Verlag Berlin, Heidelberg (2007).

25. Zhang, Z.: Microsoft Kinect sensor and its effect. IEEE Multi Med. 19(2), 4-10 (2012).

26. Shotton, J., Fitzgibbon, A., Cook, M., Sharp, T., Finocchio, M., Moore, R., Kipman, A., Blake, A.: Real-time human pose recognition in parts from single depth images. In: The IEEE Computer Vision and Pattern Recognition. pp. 116-124. ACM New York, NY, USA (2011).

27. Martín-SanJosé, J.F., Juan, M.C., Gil-Gómez, J.A., Rando, N.: Flexible learning itinerary vs. linear learning itinerary. Science of Computer Programming. 88, 3-21 (2014).

28. Martín-SanJosé, J.F., Juan, M.C., Torres, E., Vicent, M.J.: Playful interaction for learning collaboratively and individually. Journal of Ambient Intelligence and Smart Environments. 6, 295-311 (2014).

29. Martín-SanJosé, J.F., Juan, M.C., Mollá, R., Vivó, R.: Advanced displays and natural user interfaces to support learning. Interactive Learning Environments. 25(1), 17-34 (2017).

30. Rodríguez-Andrés, D., Juan, M. C., Mollá, R., Méndez-López, M.: A 3D serious game for dental learning in higher education. In: Proceedings of the 17th IEEE International Conference on Advanced Learning Technologies (ICALT2017). pp. 111-115. IEEE (2017).

31. Homer, B., Kinzer, C., Plass, J., Letourneau, S., Hoffman, D., Bromley, M., Hayward, E., Turkay, S., Kornak, Y.: Moved to learn: The effects of interactivity in a Kinect-based literacy game for beginning readers. Computers \& Education. 74, 37-49 (2014).

32. Lin, J., Sun, Q., Li, G., He, Y.: SnapBlocks: A snapping interface for assembling toy blocks with XBOX Kinect. Multimed. Tools Appl. 73, 2009-2032 (2014). 
33. Sun, C., Zhang, T., Bao, B.K., Xu, C., Mei, T.: Discriminative exemplar coding for sign language recognition with Kinect. IEEE Trans. Cybern. 43, 1418-1428 (2013).

34. Lee, G.C., Yeh, F.-H., Hsiao, Y.-H.: Kinect-based Taiwanese sign-language recognition system. Multimed. Tools Appl. 75, 261-279 (2016).

35. Armin, K., Mehrana, Z., Fatemeh, D.: Using Kinect in teaching children with hearing and visual impairment. In: Proceedings of the 4th International Conference on e-Learning and e-Teaching (ICELET 2013). pp. 86-90. IEEE (2013).

36. Retalis, S., Boloudakis, M., Altanis, G., Nikou, N.: Children with Motor Impairments Play a Kinect Learning Game: First Findings from a Pilot Case in an Authentic Classroom Environment. Interact. Des. Archit. 19, 91-104 (2014).

37. Luna-Oliva, L., Ortiz-Gutiérrez, R.M., Cano-de la Cuerda, R., Piédrola, R.M., AlguacilDiego, I.M., Sánchez-Camarero, C., Martínez Culebras, M.D.C.: Kinect Xbox 360 as a therapeutic modality for children with cerebral palsy in a school environment: a preliminary study. NeuroRehabilitation 33, 513-521 (2013).

38. Jordan, K., King, M., Hellersteth, S., Wirén, A., Mulligan, H.: Feasibility of using a humanoid robot for enhancing attention and social skills in adolescents with autism spectrum disorder. Int. J. Rehabil. Res. 36, 221-227 (2013).

39. Moriguchi, Y., Kanda, T., Ishiguro, H., Shimada, Y., Itakura, S.: Can young children learn words from a robot?. Interact. Stud. 12, 107-118 (2011).

40. Movellan, J.R., Eckhardt, M., Virnes, M., Rodriguez, A.: Sociable robot improves toddler vocabulary skills. In: Proceedings of the 4th ACM/IEEE Int. Conf. Hum. Robot Interact. pp. 307-308. ACM New York, NY, USA (2009).

41. Keren, G., Fridin, M.: Kindergarten Social Assistive Robot (KindSAR) for children's geometric thinking and metacognitive development in preschool education: A pilot study. Comput. Human Behav. 35, 400-412 (2014).

42. Keren, G., Ben-David, A., Fridin, M.: Kindergarten assistive robotics (KAR) as a tool for spatial cognition development in pre-school education. In: 2012 IEEE/RSJ International Conference on Intelligent Robots and Systems. pp. 1084-1089. IEEE (2012).

43. Timms, M.J.: Letting Artificial Intelligence in Education out of the Box: Educational Cobots and Smart Classrooms. Int. J. Artif. Intell. Educ. 26, 701-712 (2016).

44. Randell, C., Price, S., Rogers, Y., Harris, E., Fitzpatrick, G.: The Ambient Horn: designing a novel audio-based learning experience. Pers. Ubiquitous Comput. 8, 177-183 (2004).

45. de Graaf, M.M.A., Allouch, S.B., Klamer, T.: Sharing a life with Harvey: Exploring the acceptance of and relationship-building with a social robot. Comput. Human Behav. 43, 1 $14(2015)$.

46. Juan, M.-C., Mendez-Lopez, M., Perez-Hernandez, E., Albiol-Perez, S.: Augmented Reality for the assessment of children's spatial memory in real settings. PLoS One. 9, e113751 (2014).

47. Mendez-Lopez, M., Perez-Hernandez, E., Juan, M.C.: Learning in the navigational space: Age differences in a short-term memory for objects task. Learning and individual differences. 60, 11-22 (2016).

48. Rodríguez-Andrés, D., Juan, M.-C., Méndez-López, M., Pérez-Hernández, E., Lluch, J.: MnemoCity Task: Assessment of children's spatial memory using stereoscopy and virtual environments. PLoS One. 11, e0161858 (2016).

49. Cárdenas-Delgado, S., Méndez-López, M., Juan, M.-C., Pérez-Hernández, E., Lluch, J., Vivó, R.: Using a virtual maze task to assess spatial short-term memory in adults. In: Proceedings of the International Conference on Computer Graphics Theory and Applications, pp. 46-57. SCITEPRESS (2017). 
50. Lumsden, J., Edwards, E.A., Lawrence, N.S., Coyle, D., Munafò, M.R.: Gamification of cognitive assessment and cognitive training: a systematic review of applications and efficacy. JMIR Serious Games. 4, e11 (2016).

51. Reynolds, C.R., Bigler, E.D.: TOMAL test of memory and learning: Examiner's manual. Austin, TX: Pro-Ed [TOMAL Test de memoria y aprendizaje. Manual de interpretación (E. Goikoetxea, \& Departamento I+D de TEA Ediciones, Adapters), (2001, Madrid, Spain: TEA Ediciones)] (2001)

52. Brancal, M.F., Alcantud, F., Ferrer, A.M., Quiroga, M.E.: EDAF: Evaluación de la discriminación auditiva y fonológica. TEA Ediciones, Madrid, Spain: Lebón (2009).

53. Kamphaus, K.W., Perez-Hernandez, E., Sanchez-Sanchez, F.: Cuestionario de Evaluación Clínica de la Memoria. In press. Madrid: TEA Ediciones.

54. Lewis, J. R.: IBM computer usability satisfaction questionnaires: Psychometric evaluation and instructions for use. International Journal of Human-Computer Interaction. 7(1), 57-78 (1995).

55. Lund, A.M.: Measuring Usability with the USE Questionnaire. Usability User Exp. Newslett. STC Usability SIG. 8(2), 1-4 (2001).

56. Vallejo, V., Wyss, P., Chesham, A., Mitache, A.V., Müri, R.M., Mosimann, U.P., Nef, T.: Evaluation of a new serious game based multitasking assessment tool for cognition and activities of daily living: Comparison with a real cooking task. Comput. Human Behav. 70, 500-506 (2017).

57. Tarnanas, I., Tsolaki, M., Nef, T.M., Müri, R., Mosimann, U.P.: Can a novel computerized cognitive screening test provide additional information for early detection of Alzheimer's disease?. Alzheimer's \& Dementia, 10, 790-798 (2014).

58. Spooner, D., Pachana, N.: Ecological validity in neuropsychological assessment: A case for greater consideration in research with neurologically intact populations. Arch. Clin. Neuropsychol. 21, 327-337 (2006).

59. Tarnanas, I., Schlee, W., Tsolaki, M., Müri, R., Mosimann, U., Nef, T.: Ecological validity of virtual reality daily living activities screening for early dementia: Longitudinal study. JMIR Serious Games. 1, e1 (2013). 